\title{
Theoretical prediction of spectral and optical properties of bacteriochlorophylls in thermally disordered LH2 antenna complexes
}

\author{
Lorant Janosi and loan Kosztin ${ }^{\text {a) }}$ \\ Department of Physics and Astronomy, University of Missouri, Columbia, Missouri 65211 \\ Ana Damjanović \\ Department of Biophysics, The Johns Hopkins University, Baltimore, Maryland 21218
}

(Received 7 February 2006; accepted 12 May 2006; published online 5 July 2006)

\begin{abstract}
A general approach for calculating spectral and optical properties of pigment-protein complexes of known atomic structure is presented. The method, that combines molecular dynamics simulations, quantum chemistry calculations, and statistical mechanical modeling, is demonstrated by calculating the absorption and circular dichroism spectra of the B800-B850 bacteriochlorophylls of the LH2 antenna complex from Rs. molischianum at room temperature. The calculated spectra are found to be in good agreement with the available experimental results. The calculations reveal that the broadening of the B800 band is mainly caused by the interactions with the polar protein environment, while the broadening of the B850 band is due to the excitonic interactions. Since it contains no fitting parameters, in principle, the proposed method can be used to predict optical spectra of arbitrary pigment-protein complexes of known structure. (C) 2006 American Institute of Physics. [DOI: 10.1063/1.2210481]
\end{abstract}

\section{INTRODUCTION}

Pigment-protein complexes (PPCs) play an important role in photosynthetically active biological systems and have been the subject of numerous experimental and theoretical studies. ${ }^{1}$ In a PPC the photoactive pigment molecules are held in well defined spatial configuration and orientation by a scaffold of proteins. The availability of high resolution crystal structure for a continuously growing number of PPCs provides a unique opportunity in better understanding their properties and function at atomic level. The spectral and optical properties of PPCs are determined by (i) the chemical nature of the pigment, (ii) the electronic interactions between the pigment molecules, and (iii) the interactions between pigment molecules and their environment (e.g., protein, lipid, and solvent). Since in biological systems PPCs exist and function at physiological temperature their electronic and optical properties are strongly affected by thermal fluctuations which represent the main source of dynamic disorder in these systems.

Unfortunately, even in the simplest theoretical models of PPCs the simultaneous treatment of the electronic coupling between the pigments and the effect of thermal disorder can be done only approximately. ${ }^{2-5}$ The purpose of this paper is to formulate and implement an efficient method for calculating spectral and optical properties, e.g., linear absorption [optical density (OD)] and circular dichroism (CD) spectra of PPCs at finite temperature by using only atomic structure information. To demonstrate its usefulness, we apply the proposed method to calculate the OD and CD spectra at room

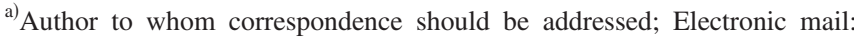
kosztini@missouri.edu
}

temperature of the aggregate of bacteriochlorophyll-a (BChl-a) molecules in the LH2 antenna complex from the purple bacterium Rs. molischianum. Following their crystal structure determination, LH2 complexes from Rs. molischianum ${ }^{6}$ and Rsp. acidophila ${ }^{7}$ have been extensively studied both experimentally ${ }^{8-15}$ and theoretically. ${ }^{8-10,16-23}$ In Rs. molischianum the LH2 is an octamer of $\alpha \beta$ heterodimers arranged in a ringlike structure ${ }^{24,25}$ (Fig. 1). Each protomer

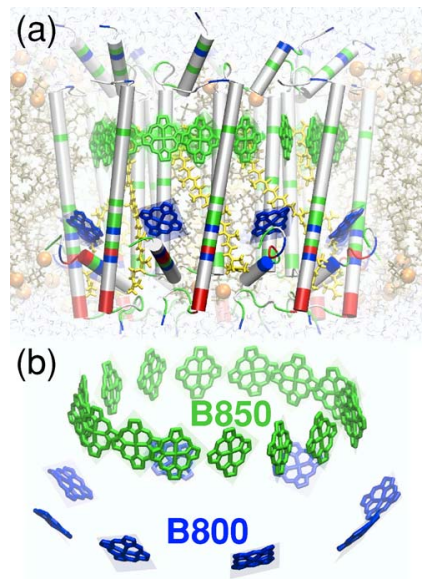

FIG. 1. (Color online) (a) Side view of an octameric LH2 complex from Rs. molischianum embeded in a fully solvated POPC lipid bilayer. The transmembrane helices of the apoprotein subunits are shown as cylinders (cartoon representation) and are colored by residue type; dark (light) colors represent hydrophilic (hydrophobic) residues. For clarity only the BChl marcrocycles are shown and the front half of the lipids are not shown. The clearly visible B800 (B850) ring is surrounded mostly by polar and charged (nonpolar) protein residues. (b) Tilted side view of the quantum system formed by the optically active B800 and B850 macrocycles that form rings oriented parallel to the surface of the membrane. [Figures rendered with the program VMD (Ref. 33).] 
consists of an $\alpha$ and a $\beta$ apoprotein which binds noncovalently one BChl-a molecule that absorbs at $800 \mathrm{~nm}$ (referred to as B800), two BChl-a molecules that absorb at $850 \mathrm{~nm}$ (referred to as B850), and at least one carotenoid that absorbs around $500 \mathrm{~nm}$. The total of $16 \mathrm{~B} 850$ and 8 B800 BChls form two circular aggregates, both oriented parallel to the surface of the membrane. The excitonic coupling between the B800s is negligible because of their large spatial separation $(\sim 22 \AA)$. Therefore, the optically active $Q_{y}$ excited electronic states of the B800s are almost degenerate. On the other hand, the tightly packed B850s (with and average $\mathrm{Mg}-\mathrm{Mg}$ distance of $\sim 9.2 \AA$ within the $\alpha \beta$ heterodimer and $\sim 8.9 \AA$ between the neighboring protomers) are strongly coupled and the corresponding $Q_{y}$ excited states form an excitonic band in which the states that carry most of the oscillator strength are clustered about $\sim 850 \mathrm{~nm}$ $(1.46 \mathrm{eV})$. Another important difference between the two $\mathrm{BChl}$ rings is that while the B800s are surrounded by mostly hydrophilic protein residues the binding pocket of the B850s is predominantly hydrophobic. ${ }^{6}$ Thus, although both B800s and B850s are chemically identical BChl-a molecules their specific spatial arrangement and the nature of their immediate protein surrounding alter differently their spectral and optical properties. For example, it is quite surprising that the two peaks, due to the B800 and B850 BChls, in the experimental OD spectrum of LH2 from Rs. molischianum at room temperature $^{19,26}$ have comparable widths although, as mentioned above, the B800 levels are almost degenerate while the B850 levels form a $\sim 0.2 \mathrm{eV}$ wide excitonic band.

Clearly, novel methods for calculating optical spectra of PPCs by using computer simulations based entirely on the atomic structure of the system would not only provide a better understanding and interpretation of the existing experimental results but would also help in predicting and designing new experiments. The standard procedure to simulate the experimental spectra of LH2 systems (and PPCs in general) consists of two steps. ${ }^{3,4,27}$ First, the excitation energy spectrum is determined based on the static crystal structure of the system and, second, the corresponding stick spectrum is "dressed up" with simulated Gaussian (in case of static disorder) and/or Lorentzian (in case of dynamic disorder) linewidths characterized by empirically (and often self consistently) determined parameters. Alternatively, the spectral broadening of the stick spectrum can also be described through the coupling of the electronic excitations (modeled as two or multilevel systems) to a stochastic heat bath characterized by a model spectral density with empirical parameters. While either approach may yield excellent agreement between the simulated and experimental spectra, the empirical nature of the model parameters restricts their predictive power.

Our proposed method for calculating optical spectra is based on a combination of all atom molecular dynamics (MD) simulations, quantum chemistry (QC) calculations, and quantum many-body theory. The conformational dynamics of the LH2 ring embedded into its natural environment (a fully solvated lipid bilayer) are followed by means of classical MD simulations. Next, for each BChl, modeled as a quantum two-level system, the $Q_{y}$ excitation energy gap and transition dipole moment time series are determined along a properly chosen segment of the MD trajectory by means of QC calculations. Finally, the OD and CD spectra are determined as weighted sums of the Fourier transform of the quantum dipole-dipole correlation function (i.e., the absorption line shape function) which, within the cumulant approximation, can be calculated from the sole knowledge of the energy gap time series. Formally, this method can also be regarded as a two step procedure. First, a stick spectrum is generated from the average values of the energy gap time series and, second, spectral broadening is applied through the corresponding line shape function weighted by the mean transition dipole (rotational) strength in the case of OD (CD) spectrum. Since both the peak position and the broadening of the optical spectrum are obtained from the same energy gap time series determined from combined MD/QC calculations, the proposed method requires no empirical fitting parameters making it ideal for predicting optical spectra for PPCs with known structure. Similar MD/QC methods were used previously by Mercer et $a l^{28}$ to calculate the OD spectrum of BChl-a in methanol, and by Damjanovic et al. ${ }^{16}$ to determine the OD and CD spectra of B850s in LH2 from Rs. molischianum. The relationship between these studies and the present one will be established below.

The reminder of this paper is organized as follows. The theoretical background of the proposed method for calculating optical spectra of PPCs is presented in Sec. II. The employed MC simulations and QC calculations are described in Sec. III. The obtained results and their discussion are contained in Sec. IV. Finally, Sec. V is reserved for conclusions.

\section{THEORY}

In order to calculate the linear optical absorption of a PPC we assume that the electronic properties of individual pigment molecules can be described in terms of a two-level system, formed by the ground state and the lowest excited singlet state (e.g., the $Q_{y}$ state in the case of BChl-a) involved in the optical absorption process. Neglecting for the moment the direct interaction between the pigments (e.g., by assuming a sufficiently large spatial separation between them as in the case of the B800s in LH2), we denote these two states for the $n$th pigment $(n=1, \ldots, N)$ as $|0\rangle \equiv\left|0_{n}\right\rangle$ and $|n\rangle$ $\equiv\left|1_{n}\right\rangle$, respectively. Once the interaction between the pigment and its environment (composed of protein matrix, lipid membrane, and solvent molecules) is taken into account these two levels turn into, still well separated, energy bands $\left|0 ; \lambda_{0}\right\rangle=|0\rangle\left|\lambda_{0}\right\rangle$ and $\left|n ; \lambda_{n}\right\rangle=|n\rangle\left|\lambda_{n}\right\rangle$, where the quantum numbers $\lambda_{0}$ and $\lambda_{n}$ specify the state of the $n$th pigment on the ground- and excited-state potential energy surface, respectively. Because the exact quantum mechanical treatment of the eigenstates $\left|0 ; \lambda_{0}\right\rangle,\left|n ; \lambda_{n}\right\rangle$, and of the corresponding energy eigenvalues $\mathcal{E}_{0, \lambda_{0}}, \mathcal{E}_{n, \lambda_{n}}$ is not feasible, usually the quantum numbers $\lambda_{0}$ and $\lambda_{n}$ are associated with the vibronic states of the PPC that can be treated within the harmonic approximation. Here we follow a different approach in which the dynamics of the nuclear degrees of freedom of the PPC are described by all atom MD simulations, and the energy gap time series $\Delta E_{n}(t)=\mathcal{E}_{n}(t)-\mathcal{E}_{0}(t)$ is calculated at each MD 
time step by QC calculations as described below. The main assumption of this approach is that the obtained energy gap time series $\Delta E_{n}(t)$ can be used to calculate approximately equilibrium quantities (such as energy gap density of states and time autocorrelation functions) of the original system without the knowledge of the exact energy gap spectrum $\Delta \mathcal{E}_{n, \lambda_{n}, \lambda_{0}}=\mathcal{E}_{n, \lambda_{n}}-\mathcal{E}_{0, \lambda_{0}}$.

In the absence of the excitonic coupling between the pigment molecules, the Hamiltonian of the system can be written as $\mathcal{H}=H_{0}+H$, where

$$
H_{0}=\sum_{\lambda_{0}}\left|0 ; \lambda_{0}\right\rangle \mathcal{E}_{0, \lambda_{0}}\left\langle 0 ; \lambda_{0}\right|
$$

and

$$
H=\sum_{n} H_{n}=\sum_{\lambda_{n}}\left|n ; \lambda_{n}\right\rangle \mathcal{E}_{n, \lambda_{n}}\left\langle n ; \lambda_{n}\right| .
$$

The dipole moment operator through which the incident light field couples to the $n$th pigment is given by

$$
\hat{\boldsymbol{\mu}}_{n}=\sum_{\lambda_{n}, \lambda_{0}} \mathbf{d}_{n, \lambda_{n}, \lambda_{0}}\left|n ; \lambda_{n}\right\rangle\left\langle 0 ; \lambda_{0}\right|,
$$

where the transition dipole moment (TDM) matrix element $\mathbf{d}_{n, \lambda_{n}, \lambda_{0}}$ in the Condon approximation ${ }^{4}$ can be written as

$$
\mathbf{d}_{n, \lambda_{n}, \lambda_{0}} \approx \mathbf{d}_{n}\left\langle\lambda_{n} \mid \lambda_{0}\right\rangle
$$

Here $\mathbf{d}_{n}=\left\langle 1\left|\hat{\boldsymbol{\mu}}_{n}\right| 0\right\rangle$ is the real TDM vector whose time series can be determined from the same combined MD/QC calculations as $\Delta E_{n}(t)$. Note that while $\langle 1 \mid 0\rangle=0$, in general the Franck-Condon factors $\left\langle\lambda_{n} \mid \lambda_{0}\right\rangle$ are finite. ${ }^{4}$

When the size of the PPC is much smaller than the wavelength of the light field, in leading approximation the latter can be regarded as homogeneous throughout the system and, according to standard linear response theory, the corresponding OD spectrum is proportional to the dipoledipole correlation function,

$$
I(\omega) \propto \omega \sum_{n, m} \operatorname{Re}\left[\int_{0}^{\infty} d t e^{i \omega t}\left\langle\hat{\mu}_{m, i}^{\dagger}(0) \hat{\mu}_{n, i}(t)\right\rangle\right],
$$

where $\hat{\mu}_{n, i}(t)=e^{-i H t} \hat{\mu}_{n, i}(0) e^{i H_{0} t}$ is the $i \in\{x, y, z\}$ component of the time dependent electric dipole operator, and $\langle\cdots\rangle$ $=\operatorname{Tr}\left\{Z_{0}^{-1} \exp \left(-\beta H_{0}\right) \cdots\right\}$ with $\beta=1 / k_{B} T$ the usual temperature factor and $Z_{0}$ the corresponding partition function. To simplify the notation, throughout this paper we use units in which $\hbar=1$, and apply the convention of implicit summation over repeated vector indices. By employing Eqs. (1) and (2), after some algebra, the quantum dipole correlation function in Eq. (3) can be expressed as

$$
\left\langle\hat{\mu}_{m, j}^{\dagger}(0) \hat{\mu}_{n, i}(t)\right\rangle=d_{n, i} d_{m, j} \delta_{n m}\left\langle e^{i H_{0} t} e^{-i H_{n} t}\right\rangle,
$$

where $\delta_{n m}$ is the Kronecker delta. By inserting Eq. (4) into Eq. (3) one obtains the sought OD spectrum of an aggregate of noninteracting pigments in their native environment,

$$
I(\omega) \propto \omega \sum_{n} d_{n}^{2} A_{n}(\omega),
$$

where the line shape function is defined as

$$
A_{n}(\omega)=\operatorname{Re} \int_{0}^{\infty} d t e^{i \omega t}\left\langle e^{i H_{0} t} e^{-i H_{n} t}\right\rangle .
$$

The main difficulty in calculating the quantum time correlation function in Eq. (5b) is due to the fact that the Hamiltonians $H_{0}$ and $H_{n}$ do not commute. If they would, then the line shape function could be expressed in terms of the energy gap density of states (DOS). Indeed, in this case $\left\langle e^{i H_{0} t} e^{-i H_{n} t}\right\rangle \approx\left\langle\exp \left(-i \Delta H_{n} t\right)\right\rangle$, with $\Delta H_{n}=H_{n}-H_{0}$, and by calculating the time integral in Eq. (5b) would follow

$$
\begin{aligned}
& A_{n}(\omega) \approx \pi \mathcal{N}(\omega), \\
& \mathcal{N}(\omega) \equiv\left\langle\delta\left(\omega-\Delta H_{n}\right)\right\rangle \approx\left\langle\delta\left(\omega-\Delta E_{n}(t)\right)\right\rangle,
\end{aligned}
$$

where the density of states $\mathcal{N}(\omega)$ is approximated by the binned histogram of the energy gap fluctuations $\Delta E_{n}(t)$ obtained from combined MD/QC calculations. In general, Eqs. (6) overestimate the broadening of the line shape function. Indeed, the Fourier transform of the exact spectral representation of the correlation function,

$$
\left\langle e^{-i H_{0} t} e^{i H t}\right\rangle=\sum_{\lambda_{0}, \lambda_{n}} \rho_{\lambda_{0}}\left|\left\langle\lambda_{0} \mid \lambda_{n}\right\rangle\right|^{2} e^{-i\left(\mathcal{E}_{n, \lambda_{n}}-\mathcal{E}_{0, \lambda_{0}}\right) t},
$$

where $\rho_{\lambda_{0}}=Z_{0}^{-1} \exp \left(-\beta \mathcal{E}_{0, \lambda_{0}}\right)$ is the statistical matrix of the electronic ground state, yields

$$
A(\omega)=2 \pi \sum_{\lambda_{0}, \lambda_{n}} \rho_{\lambda_{0}}\left|\left\langle\lambda_{0} \mid \lambda_{n}\right\rangle\right|^{2} \delta\left(\omega-\Delta \mathcal{E}_{n, \lambda_{n}, \lambda_{0}}\right),
$$

which can be regarded as a Franck-Condon weighted and thermally averaged density of states. ${ }^{29}$ By setting the FranckCondon factors $\left\langle\lambda_{0} \mid \lambda_{n}\right\rangle$ equal to unity in (7b) one obtains Eqs. (6). Since it is not possible to determine all these factors, it is often convenient to use Eqs. (6) as a rough estimate of $A_{n}(\omega)$ for calculating the OD spectrum.

A systematic way of calculating the correlation function in $(5 b)$ is the cumulant expansion method. Here we employ the second order cumulant approximation that is often used in optical spectra calculations. ${ }^{3}$ We have

$$
\begin{aligned}
\left\langle e^{i H_{0} t} e^{-i H_{n} t}\right\rangle & =\left\langle T \exp \left[-i \int_{0}^{t} d \tau \Delta H_{n}(\tau)\right]\right\rangle \\
& \approx \exp \left[-i\left\langle\Delta H_{n}\right\rangle t-\int_{0}^{t} d \tau(t-\tau) \mathcal{C}_{n}(\tau)\right],
\end{aligned}
$$

where $T$ is the time ordering operator, $\Delta H_{n}(t)$ $=e^{i H_{0} t} \Delta H_{n} e^{-i H_{0} t}, \mathcal{C}_{n}(t)=\left\langle\delta H_{n}(t) \delta H_{n}(0)\right\rangle$, and $\delta H_{n}(t)=\Delta H_{n}(t)$ $-\left\langle\Delta H_{n}\right\rangle$. To make progress, the quantum statistical averages in Eq. (8) will be approximated with classical ones involving the energy gap time series $\Delta E_{n}(t)$, i.e.,

$$
\begin{aligned}
& \left\langle\Delta H_{n}\right\rangle \approx\left\langle\Delta E_{n}(t)\right\rangle \equiv \omega_{n}, \\
& \operatorname{Re}\left[\mathcal{C}_{n}(t)\right] \approx C_{n}(t) \equiv\left\langle\delta E_{n}(t) \delta E_{n}(0)\right\rangle,
\end{aligned}
$$

where $\delta E_{n}(t)=\Delta E_{n}(t)-\left\langle\Delta E_{n}\right\rangle$. While approximating a quantum time correlation function by identifying its real part with the corresponding classical correlation function as in Eq. (9b) is widely used, ${ }^{28,30,31}$ other approximation schemes have also been considered in the literature. ${ }^{32}$ Next, by invoking 
the fluctuation dissipation theorem $\widetilde{\mathcal{C}}_{n}(-\omega)=\exp (-\beta \omega) \widetilde{\mathcal{C}}_{n}(\omega)$, where $\widetilde{\mathcal{C}}_{n}(\omega)=\int_{-\infty}^{\infty} d t \mathcal{C}_{n}(t) \exp (i \omega t)$ is the Fourier transform of $\mathcal{C}_{n}(t)$, the quantum correlation function in terms of the real spectral density,

$$
J_{n}(\omega)=\frac{1}{2}\left[\widetilde{\mathcal{C}}_{n}(\omega)-\widetilde{\mathcal{C}}_{n}(-\omega)\right]=\frac{1}{2}\left(1-e^{-\beta \omega}\right) \widetilde{\mathcal{C}}_{n}(\omega),
$$

can be written as

$$
\begin{aligned}
\mathcal{C}_{n}(t) & =\mathcal{C}_{n}^{\prime}(t)-i \mathcal{C}_{n}^{\prime \prime}(t) \\
& =\int_{0}^{\infty} \frac{d \omega}{\pi} J_{n}(\omega)[\operatorname{coth}(\beta \omega / 2) \cos \omega t-i \sin \omega t] .
\end{aligned}
$$

By identifying the real part of Eq. (11) with Eq. (9b) one can determine both the spectral density and the imaginary part of the quantum correlation function, i.e.,

$$
J_{n}(\omega)=2 \tanh (\beta \omega / 2) \int_{0}^{\infty} d t C_{n}(t) \cos \omega t,
$$

and

$$
\mathcal{C}_{n}^{\prime \prime}(t)=\int_{0}^{\infty} \frac{d \omega}{\pi} J_{n}(\omega) \sin \omega t .
$$

Thus, the line shape function within the second cumulant approximation is

$$
\begin{aligned}
A_{n}(\omega) & \equiv \bar{A}_{n}\left(\omega-\omega_{n}\right) \\
& =\int_{0}^{\infty} d t e^{-\phi_{n}(t)} \cos \left[\left(\omega-\omega_{n}\right) t+\varphi_{n}(t)\right],
\end{aligned}
$$

where the broadening and frequency shift functions are given by

$$
\phi_{n}(t)=\int_{0}^{t} d \tau(t-\tau) C_{n}(\tau),
$$

and

$$
\varphi_{n}(t)=\int_{0}^{\infty} \frac{d \omega}{\pi} J_{n}(\omega) \frac{\omega t-\sin \omega t}{\omega^{2}} .
$$

A straightforward extension of the above method for calculating the line shape function and the OD spectrum of $N$ excitonically coupled pigment molecules would require the determination of the energies $\mathcal{E}_{J, \lambda_{J}}$ and TDMs $\mathbf{d}_{J}$ corresponding to the excitonic states $\left|J ; \lambda_{J}\right\rangle, J=1, \ldots, N$. Unfortunately, the required QC calculations (by considering all $N$ pigments as a single quantum system) are still prohibitively expensive computationally. Therefore, we employed an effective Hamiltonian approximation for determining the time series $\Delta E_{J}(t)=\mathcal{E}_{J}(t)-\mathcal{E}_{0}(t)$ and $\mathbf{d}_{J}(t)$ from $\Delta E_{n}(t)$ and $\mathbf{d}_{n}(t)$ of the individual pigments. Assuming that these are coupled through the usual point dipole-dipole interaction,

$$
V_{n m}=\frac{1}{4 \pi \varepsilon_{0} \varepsilon_{r}}\left[\frac{\mathbf{d}_{n} \mathbf{d}_{m}}{r_{n m}^{3}}-3 \frac{\left(\mathbf{d}_{n} \cdot \mathbf{r}_{n m}\right)\left(\mathbf{d}_{m} \cdot \mathbf{r}_{n m}\right)}{r_{n m}^{5}}\right],
$$

where $\varepsilon_{r}$ is the relative dielectric permitivity of the medium, $\mathbf{r}_{n}$ is the position vector of pigment $n$, and $\mathbf{r}_{n m}=\mathbf{r}_{m}-\mathbf{r}_{n}$, the eigenvalue equation one needs to solve at every MD time step is

$$
\sum_{m}\left[\left(\Delta E_{n} \delta_{n m}+V_{n m}\right)-\Delta E_{J} \delta_{n m}\right] c_{m}^{(J)}=0 .
$$

In terms of the coefficients $c_{n}^{(J)}=\langle J \mid n\rangle$ the excitonic TDMs are

$$
\mathbf{d}_{J}=\sum_{n}\langle J \mid n\rangle \mathbf{d}_{n} .
$$

Next, by rewriting the Hamiltonian (1b) in diagonal form (i.e., in terms of noninteracting excitons) $H=\Sigma_{J} H_{J}$ $=\Sigma_{J, \lambda_{J}}\left|J ; \lambda_{J}\right\rangle \mathcal{E}_{J, \lambda_{J}}\left\langle J ; \lambda_{J}\right|$, after some algebra one arrives at the equations

$$
\left\langle\hat{\mu}_{m, j}^{\dagger}(0) \hat{\mu}_{n, i}(t)\right\rangle=\sum_{J}\langle J \mid n\rangle d_{n, i} d_{m, j}\langle m \mid J\rangle\left\langle e^{i H_{0} t} e^{-i H_{J} t}\right\rangle,
$$

and

$$
\sum_{n, m}\left\langle\hat{\mu}_{m, j}^{\dagger}(0) \hat{\mu}_{n, i}(t)\right\rangle=\sum_{J} d_{J, i} d_{J, j}\left\langle e^{i H_{0} t} e^{-i H_{J} t}\right\rangle .
$$

Inserting Eq. (18b) into Eq. (3) one obtains the desired OD spectrum of the excitonic system,

$$
I(\omega) \propto \omega \sum_{J} d_{J}^{2} A_{J}(\omega),
$$

where

$$
A_{J}(\omega)=\operatorname{Re} \int_{0}^{\infty} d t e^{i \omega t}\left\langle e^{i H_{0} t} e^{-i H_{J} t}\right\rangle .
$$

We note that by replacing the site index $n$ with the excitonic index $J$ most of the above results for noninteracting pigments remain formally valid for the corresponding excitonic system as well. For example, similar expressions to (6) and (14) can be easily derived for estimating $A_{J}(\omega)$.

To conclude this section we derive an expression for the $\mathrm{CD}$ spectrum of the PPC. By definition, the CD spectrum $I_{\mathrm{CD}}(\omega)$ is the difference between $I_{L}(\omega)$ and $I_{R}(\omega)$, the OD spectra for left and right circularly polarized light, respectively. Unlike in the case of the OD spectrum, the calculation of $I_{\mathrm{CD}}(\omega)$ even within the leading order approximation requires taking into account the spatial variation of the light field across the PPC as well as the excitonic coupling between the pigment molecules regardless how small this may be. The sensitivity of the $\mathrm{CD}$ spectrum to geometrical and local details of the PPC makes it a quantity difficult to predict by theoretical modeling. The $\mathrm{CD}$ spectrum is given by ${ }^{5}$

$$
\begin{aligned}
I_{\mathrm{CD}}(\omega)= & \frac{1}{4}\left[I_{L}(\omega)-I_{R}(\omega)\right] \propto \omega \operatorname{Re} \int_{0}^{\infty} d t e^{i \omega t} \\
& \times \sum_{n, m} \frac{\pi}{\lambda} \varepsilon_{i j k}\left(\mathbf{r}_{n}\right)_{k}\left\langle\hat{\mu}_{m, i}^{\dagger}(0) \hat{\mu}_{n, i}(t)\right\rangle,
\end{aligned}
$$

where $\lambda$ is the wavelength of the incident light and $\epsilon_{i j k}$ is the unit antisymmetric tensor of rank 3. Inserting Eq. (18a) into (21) and making use of Eq. (20), we obtain 


$$
I_{\mathrm{CD}}(\omega) \propto \omega \sum_{J} R_{J} A_{J}(\omega),
$$

where

$$
R_{J}=\frac{\pi}{\lambda} \sum_{n, m}\langle J \mid n\rangle\left[\mathbf{r}_{n} \cdot\left(\mathbf{d}_{n} \times \mathbf{d}_{m}\right)\right]\langle m \mid J\rangle
$$

is the so-called rotational strength of the excitonic state $J$. Note that in the absence of the excitonic coupling all $R_{J}=0$ (because for a given $J$ only one coefficient $\langle J \mid n\rangle$ is nonzero) and the CD spectrum vanishes. The rotational strength plays the same role for the CD spectrum as the TDM strength for the OD spectrum. Specifically, $R_{J}$ gives the coupling between the TDM of the excitonic state $J$ and the orbital magnetic moment of the other excitons. The coupling to the local magnetic moment is assumed to be small (Cotton effect) and usually is discarded. ${ }^{14,27}$

\section{COMPUTATIONAL METHODS}

In order to apply the results derived in Sec. II or calculating the OD and CD spectra of the B800 and B850 BChls in a single LH2 ring from Rs. molischianum, first we need to determine the time series of the $Q_{y}$ energy gap $\Delta E_{n}(\ell \Delta t)$ and TDM $\mathbf{d}_{n}(\ell \Delta t), \ell=0,1, \ldots, N_{t}$, for all individual BChls. We accomplish this in two steps. First, we use all atom MD simulations to follow the dynamics of the nuclear degrees of freedom by recording snapshots of the atomic coordinates at times $t_{\ell}=\ell \Delta t$, and then use QC calculations to compute $\Delta E_{n}$ and $\mathbf{d}_{n}$ for each snapshot.

\section{A. Molecular dynamics simulations}

Here we provide a brief description of the simulated $\mathrm{LH} 2$ ring in its native environment as well as the employed MD simulation protocol. A more detailed account of the reported MD simulations can be found in Ref. 16. A perfect eightfold LH2 ring (Fig. 1) was constructed starting from the crystal structure [protein data bank (PDB) code $1 \mathrm{LGH}$ ] of Rs. molischianum]. ${ }^{6}$ After adding the missing hydrogens, the protein system was embedded in a fully solvated palmitoyloleoylphosphatidylcholine (POPC) lipid bilayer of hexagonal shape. Finally, a total of $16 \mathrm{Cl}^{-}$counterions were properly added to ensure electroneutrality of the entire system of 87055 atoms. In order to reduce the finite-size effects, the hexagonal unit cell (with side length of $\sim 60 \AA$, lipid bilayer thickness of $\sim 42 \AA$, and two water layers of combined thickness of $\sim 35 \AA$ ) was replicated in space by using periodic boundary conditions. The CHARMM27 force field parameters for proteins ${ }^{34,35}$ and lipids ${ }^{36}$ were used. Water molecules were modeled as TIP3P. ${ }^{37}$ The force field parameters for BChls and lycopenes were the ones used in Ref. 16. After energy minimization, the system was subjected to a 2 ns long equilibration in the NPT ensemble ${ }^{38}$ at normal temperature $(T=300 \mathrm{~K})$ and pressure $(p=1 \mathrm{~atm})$, using periodic boundary conditions and treating the full long-range electrostatic interactions by the particle mesh Ewald (PME) method. ${ }^{39}$ All MD simulations were preformed with the program NAMD $2.5,{ }^{40}$ with a performance of $\sim 8.5$ days $/ \mathrm{ns}$ on 24 CPUs of an AMD 1800+ Beowulf cluster. During equilibration an inte- gration time step of 2 fs was employed by using the SHAKE constraint on all hydrogen atoms. ${ }^{41}$ After the 2 ns equilibration a 1 ps production run with 1 fs integration step was carried out with atomic coordinates saved every other time step, resulting in $N_{t}=500 \mathrm{MD}$ snapshots with $\Delta t=2$ fs time separation. These configuration snapshots were used as input for the QC calculations described below.

\section{B. Quantum chemistry calculations}

The time series of the $Q_{y}$ transition energies $\Delta E_{n}$ and dipole moments $\mathbf{d}_{n}$ of individual BChls can be determined only approximately from the configuration snapshots obtained from MD simulations. The level of approximation used is determined by (i) the actual definition of the optically active quantum system, i.e., the part of the system that is responsible for light absorption and needs to be treated quantum mechanically, (ii) the actual choice of the QC method used in the calculations, and (iii) the particular way in which the effect of the (classical) environment on the quantum system is taken into account in the QC calculations. Because the optical properties of BChls are determined by the cyclic conjugated $\pi$-electron system of the macrocycle, the quantum system was restricted to a truncated structure of the BChl-a containing 49 atoms in the porphyrin plane. The truncation consisted in removing the phytyl tail and in replacing the terminal $\mathrm{CH}_{3}$ and $\mathrm{CH}_{2} \mathrm{CH}_{3}$ groups on the macrocycle with $\mathrm{H}$ atoms in order to satisfy valence requirements. Similar truncation schemes have been employed previously. ${ }^{28,42}$ In these studies the phytyl tail of the BChls was removed but the number of atoms retained in the optically active macrocycle was different. For example, Cory et al., ${ }^{42}$ in calculating the excited states of the B800 octamer and the B850 hexadecamer of LH2 from Rs. molischianum, used 44 macrocycle atoms, while Mercer et al. ${ }^{28}$ in calculating the absorption spectrum of BChl-a in ethanol, used 84 atoms. According to the crystal structure, ${ }^{6}$ the B800 and B850 BChls in LH2 from Rs. molischianum differ only in the length of their phytyl chain, having a total of 107 and 140 atoms, respectively. The removal of the phytyl tail reduces dramatically both the size of the quantum system and the corresponding QC computational time. Furthermore, for the truncated BChls the nontrivial task of automatic identification of the $Q_{y}$ excited state in the case of a large number of such computations becomes easier and more precise. Although in general the different truncation schemes yield excitation energy time series with somewhat different (shifted) mean values, the corresponding energy fluctuations, which play the chief role in calculating the optical absorption properties of PPC at room temperature in their native environment, are less sensitive to the actual size of the truncated pigment.

The $Q_{y}$ excitations of the truncated BChls were calculated by using Zerner's semiempirical intermediate neglect of differential overlap method parametrized for spectroscopy (ZINDO/S) within the single-point configuration interaction singles (CIS) approximation. ${ }^{43,44}$ Because it is much faster and more accurate than most of the computationally affordable $a b$ initio QC methods [e.g., the Hartree-Fock (HF) CIS method with the minimal STO- $3 \mathrm{G}^{*}$ basis set], ZINDO/S CIS 
has been extensively used in the literature to compute low lying optically allowed excited states of pigment molecules. ${ }^{19,20,45,46}$ In cases like ours, where thousands of QC calculations are required, the proper balancing between speed and accuracy is absolutely essential. To further increase the computational speed, the active orbital space for the CIS calculations was restricted to the ten highest occupied molecular orbitals (HOMOs) and the ten lowest unoccupied molecular orbitals (LUMOs). According to previous studies, ${ }^{28}$ as well as our own testings, the choice of a larger active space has negligible effect on the computed $Q_{y}$ states. Our ZINDO/S calculations were carried out with the QC program packages HYPERCHEM (Ref. 47 ) and GAUSSIAN $98 .^{48}$ In each calculation only the lowest four excited states were determined. Only in a small fraction $(<5 \%)$ of cases was the indentification of the $Q_{y}$ excited state (characterized by the largest oscillator strength and corresponding to transitions $\mathrm{HOMO} \rightarrow$ LUMO and HOMO $-1 \rightarrow$ LUMO +1$)$ problematic requiring careful inspection. We have found that even in such cases the $Q_{y}$ state had the largest projection of the TDM along the $y$ axis, determined by the NB and ND nitrogen atoms.

The effect of the environment on the quantum system was taken into account through the electric field created by the partial point charges of the environment atoms, including those $\mathrm{BChl}$ atoms that were removed during the truncation process. Thus, the dynamics of the nuclear degrees of freedom (described by MD simulation) have a twofold effect on the fluctuations of the $Q_{y}$ state, namely, they lead to (1) conformational fluctuation of the (truncated) BChls and (2) a fluctuating electric field created by the thermal motion of the corresponding atomic partial charges. In order to assess the relative importance of these two effects the time series $\Delta E_{n}(t)$ were calculated both in the the presence and in the absence of the point charges. Since the ZINDO/S implementation in GAUSSIAN 98 does not work in the presence of external point charges, these calculations were done with HYPERCHEM. The ZINDO/S calculations without point charges were carried out with both QC programs and yielded essentially the same result. For each case, we have performed a total of 12000 (500 snapshots $\times 24$ BChls) ZINDO/S calculations. On a workstation with dual $3 \mathrm{GHz}$ Xeon EM64T CPU it took $\sim 2.3 \mathrm{~min} / \mathrm{CPU}$ for each calculation with point charges, and only $\sim 0.7 \mathrm{~min} / \mathrm{CPU}$ without point charges. Thus, on a cluster of five such workstations, all $24000 \mathrm{ZINDO} / \mathrm{S}$ runs were completed in $\sim 1.9+0.6$ $=2.5$ days.

\section{RESULTS AND DISCUSSION}

The time series of the $Q_{y}$ excitation energies $\Delta E_{n}\left(t_{\ell}\right)$ and TDMs $\mathbf{d}_{n}\left(t_{\ell}\right),\left(t_{\ell}=\ell \Delta t, \ell=0, \ldots, N_{t}, N_{t}=499\right.$, and $\left.\Delta t=2 \mathrm{fs}\right)$, were computed with the ZINDO/S CIS method, described in Sec. III B, for both B850 $(n=1, \ldots, 16)$ and B800 $(n=17, \ldots, 24)$ BChls in a LH2 ring from Rs. molischianum, using snapshots from the all atom MD simulation described in Sec. III A. The calculations were done both with and without the point charges of the atoms surrounding the truncated BChls. The 1 ps long time series appear to be sufficiently

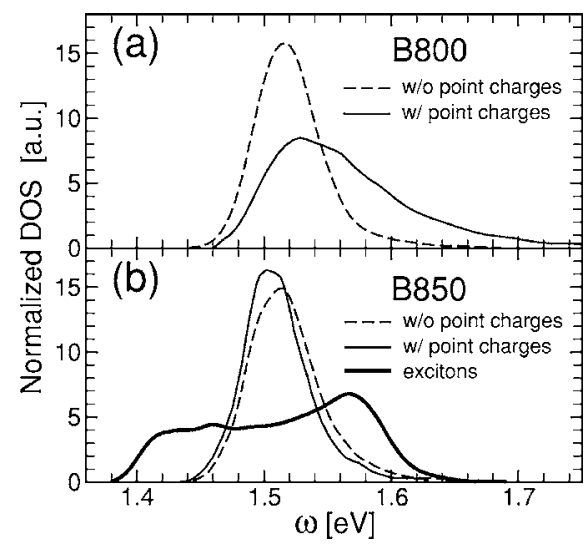

FIG. 2. Normalized DOS, $\mathcal{N}(\omega)$, for (a) B800, and (b) B850 BChls in LH2 of Rs. Molischianum computed as binned histograms of the corresponding $Q_{y}$ excitation energy time series obtained from combined MD/QC simulations. Whether the charge fluctuations of the BChls' environment are included (solid lines) or not (dashed line) makes an important difference in $\mathcal{N}(\omega)$ only for B800. In (b) the DOS of the B850 excitons is shown as a thick solid line.

long for calculating the DOS of the $Q_{y}$ excitation energies and the corresponding OD and CD spectra. In Ref. 28 it has been found that at least a 2.2 ps long MD trajectory was needed for proper evaluation of optical observables related to their MD/QC calculations. However, our test calculations showed no significant difference between the energy gap autocorrelation functions calculated from a 2 ps and a 1 ps long energy gap time series. Therefore, to reduce the computational time we have opted for the latter.

\section{A. Energy gap density of states (DOS) and transition dipole moments (TDMs)}

Figure 2 shows the $Q_{y}$ energy gap DOS, $\mathcal{N}(\omega)$, of the individual B800 [top (a) panel] and B850 [bottom (b) panel] BChls calculated, according to Eqs. (6), as normalized binned histograms of the time series $\Delta E_{\mathrm{B} 800} \equiv \Delta E_{n}\left(t_{\ell}\right)$ with $n=17, \ldots, 24$, and $\Delta E_{\mathrm{B} 850} \equiv \Delta E_{n}\left(t_{\ell}\right)$ with $n=1, \ldots, 16$, respectively. In order to eliminate the noise due to finite sampling, the graphs have been smoothened out by a running average procedure. The same smoothing out procedure has been applied to all subsequent line shape and spectra calculations. In the absence of the point charge distribution of the environment $\mathcal{N}(\omega)$ for B800 and B850 (dashed lines) are almost identical, having peak positions at $1.51 \mathrm{eV}(817 \mathrm{~nm})$ and $1.515 \mathrm{eV}(818 \mathrm{~nm})$, and full widths at half maximum (FWHMs) of 51 and $59 \mathrm{meV}$, respectively. The fact that the peak position practically coincides with the mean energy gap is indicative that the DOS is symmetric with respect to its maximum. It should be noted that essentially the same mean energy gap of $1.5 \mathrm{eV}$ was obtained in similar MD/QC calculations (i) by us (data not shown) in the case of a truncated BChl-a in vacuum but artificially coupled to a Langevin heat bath at room temperature, and (ii) by Mercer et al. ${ }^{28}$ for a BChl-a solvated in methanol also at room temperature. Although in case (ii) the width of the DOS appears to be somewhat broader $(\mathrm{FWHM} \approx 65 \mathrm{meV}$ ) than in case (i), for which $\mathrm{FWHM} \approx 58 \mathrm{meV}$, based on these results one can safely con- 
clude that the thermal motion of the nuclei in individual BChls leads to $Q_{y}$ energy gap fluctuations that are insensitive to the actual nature of the nonpolar environment. Since in LH2 from Rs. molischianum the surrounding of the B800s is polar while that of the B850s is not [see Fig. 1(a)], one expects that once the point charges of the environment are taken into account in the QC calculations $\mathcal{N}(\omega)$ should change dramatically only in the case of B800. Indeed, as shown in Fig. 2(b) (solid line), in the presence of the point charges the peak of $\mathcal{N}_{\mathrm{B} 850}(\omega)$ is only slightly redshifted to $1.502 \mathrm{eV}(825 \mathrm{~nm})$ and essentially without any change in shape with $\mathrm{FWHM} \approx 53 \mathrm{meV}$. By contrast, the DOS for B800 in the presence of the point charges [Fig. 2(a)] has qualitatively changed. The induced higher energy fluctuations not only spoil the symmetry of $\mathcal{N}_{\mathrm{B} 800}(\omega)$ but also increase dramatically its broadening, characterized by $\mathrm{FWHM} \approx 100 \mathrm{meV}$. Thus, in spite of a small blueshift to $1.528 \mathrm{eV}(811 \mathrm{~nm})$ of the peak of $\mathcal{N}_{\mathrm{B} 800}(\omega)$ the mean value of the energy gap $\left\langle\Delta E_{\mathrm{B} 800}\right\rangle=1.556 \mathrm{eV}(797 \mathrm{~nm})$ is increased considerably, matching rather well the experimental value of $800 \mathrm{~nm}$.

The time series of the excitonic energies $\Delta E_{J}\left(t_{\ell}\right), J$ $=1, \ldots, 16$, of the B850 BChls were determined by solving for each MD snapshot, within the point-dipole approximation, the eigenvalue equation (16). In calculating the matrix elements (15) $\mathbf{r}_{n}$ was identified with the position vector of the $\mathrm{Mg}$ atom in the $n$th $\mathrm{BChl}$. Consistent with the Condon approximation, the magnitude of the computed B850 TDM time series exhibited a standard deviation of less than $4 \%$ about the average value $\left\langle d_{\mathrm{B} 850}\right\rangle=11.77 \mathrm{D}$. The latter is by a factor of $k=1.87$ larger than the experimentally accepted 6.3 D value of the $Q_{y}$ TDM of BChl-a. ${ }^{49}$ Thus, to account for this overestimate of the TDM by the ZINDO/S CIS method, instead of using a reasonable value of 1.86 for the relative dielectric constant of the protein environment in Eq. (15), we set for all the calculations reported in this paper $\varepsilon_{r}$ $=6.5\left(=1.86 \times 1.87^{2}\right)$. Equivalently, one can rescale all TDMs from the ZINDO/S calculations by the factor $k^{-1}$ and set $\varepsilon_{r}$ $=1.86$. Either way the mean values of the nearest neighbor dipolar coupling energies between B850s were $27 \mathrm{meV}$ $\approx 220 \mathrm{~cm}^{-1}$ within a protomer and $24 \mathrm{meV} \approx 196 \mathrm{~cm}^{-1}$ between adjacent heterodimers. Just like in the case of individual BChls, the DOS corresponding to the B850 excitonic energies [Fig. 2(b), thick line] was calculated as a binned histogram of $\Delta E_{J}\left(t_{\ell}\right)$. As expected, the excitonic DOS is not sensitive to whether the point charges of the environment are included or not in the B850 site energy calculations.

The mean excitonic TDMs, calculated from Eq. (17) and expressed in terms of $\left\langle d_{\mathrm{B} 850}\right\rangle$, are shown in Fig. 3. The error bars represent the standard deviation of the time series $d_{J}\left(t_{\ell}\right)$. In agreement with previous studies, most of the dipole strength is amassed into the lowest three excitonic states.

As discussed in Sec. II, a rough estimate of the line shape function can be obtained as the combined DOS of the B800 BChls and B850 excitons. In this approximation the OD spectrum reads

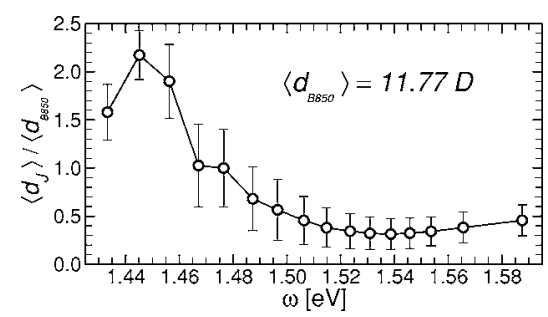

FIG. 3. Average transition dipole moments $\left\langle d_{J}\right\rangle$ corresponding to the $J$ $=1, \ldots, 16$ B850 excitonic states. Both $\left\langle d_{J}\right\rangle$ and the corresponding error bars are expressed relative to the mean dipole moment of individual B850s.

$$
\begin{array}{r}
I_{\mathrm{DOS}}(\omega) \propto \omega\left[\sum_{J} d_{J}^{2}\left\langle\delta\left(\omega-\Delta E_{J}\right)\right\rangle\right. \\
\left.+\sum_{\mathrm{B} 800} d_{\mathrm{B} 800}^{2}\left\langle\delta\left(\omega-\Delta E_{\mathrm{B} 800}\right)\right\rangle\right],
\end{array}
$$

where the B800 index in the last term means summation over all B800 BChls. Figure 4 shows the calculated $I_{\mathrm{DOS}}(\omega)$ blueshifted by $20 \mathrm{eV}$ (solid line) in order to match the B850 peak position with the one in the experimental OD spectrum $^{19,26}$ (dashed line). While the B850 band and the relative heights of the two peaks in $I_{\mathrm{DOS}}(\omega)$ match rather well the experimental data, the position and the broadening of the B800 peak do not. This result clearly shows that in general peak positions in optical spectra may be shifted from the corresponding peak positions in the excitation energy spectrum due to correlation effects between the ground and optically active excited states. The latter may also lead to different line broadenings of the corresponding peaks. Thus, it appears that, in principle, methods for simulating optical spectra in which the position of the peaks are identified with the computed excitation energies (stick spectrum) are not entirely correct and using instead more sophisticated methods that include quantum correlation effects should be preferred. Such method,

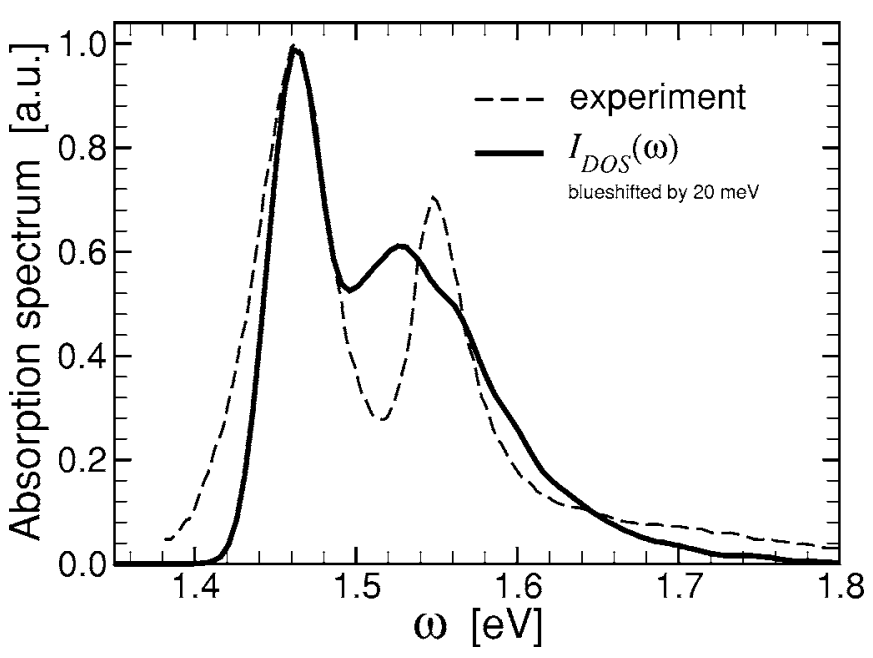

FIG. 4. Absorption spectrum $I_{\mathrm{DOS}}(\omega)$ of LH2 for Rs. molischianum calculated as a combined DOS of B800 BChls and B850 excitons weighted by the corresponding dipole strengths (solid line). $I_{\mathrm{DOS}}(\omega)$ was blueshifted by $20 \mathrm{meV}$ in order to overlay its B850 peak with the corresponding one in the experimental OD spectrum (Ref. 26) (dashed line). 


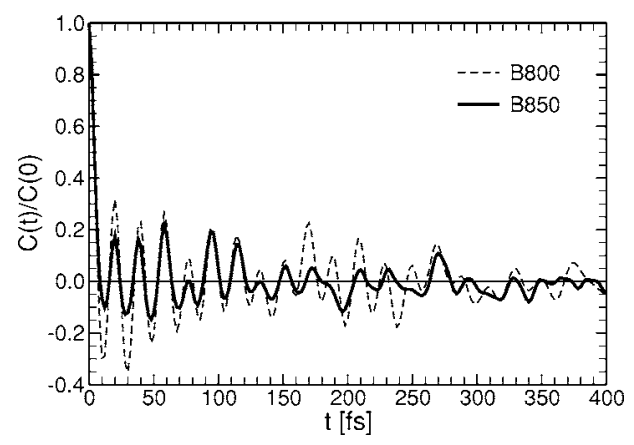

FIG. 5. Normalized autocorrelation function $C(t) / C(0)$ of the energy gap fluctuations $\delta E(t)=E(t)-\langle E\rangle$ for individual B800 (dashed line) and B850 (solid line) BChls, calculated using Eq. (24). The mean square energy gap fluctuations are $C_{\mathrm{B} 800}(0)=3.16 \times 10^{-3} \mathrm{eV}^{2}$ and $C_{\mathrm{B} 850}(0)=8.68 \times 10^{-4} \mathrm{eV}^{2}$.

based on the cumulant approximation of the line shape function as described in Sec. II, used in the next section for calculating the OD spectrum of an LH2 ring from Rs. molischianum.

\section{B. Absorption (OD) spectrum}

The key quantity for calculating the line shape functions of the individual $\mathrm{B} 850$ and $\mathrm{B} 800 \mathrm{BChls}$ is the (classical) autocorrelation function $C_{n}(t)=\left\langle\delta E_{n}(t) \delta E_{n}(0)\right\rangle$ of the energy gap fluctuation $\delta E_{n}(t)=\Delta E_{n}(t)-\left\langle\Delta E_{n}\right\rangle$ determined from the combined $\mathrm{MD} / \mathrm{QC}$ calculations. Because the time series $\Delta E_{n}(t)$ were too short for a proper evaluation of the ensemble average in the individual $C_{n}(t)$, a single time correlation function $C_{\mathrm{B} 800}(t)\left[C_{\mathrm{B} 850}(t)\right]$ was determined by averaging over all $\mathrm{B} 800$ [B850] BChls according to the formula,

$$
C_{\alpha}\left(t_{\ell}\right)=\frac{1}{M} \sum_{m}\left[\frac{1}{N_{t}-\ell} \sum_{k=1}^{N_{t}-\ell} \delta E_{m}\left(t_{\ell}+t_{k}\right) \delta E_{m}\left(t_{k}\right)\right],
$$

where $M=8$ and $m=17, \ldots, 24$ for $\alpha=\mathrm{B} 800$ and $M=16$ and $m=1, \ldots, 16$ for $\alpha=\mathrm{B} 850$. The normalized correlation functions $C_{\alpha}(t) / C_{\alpha}(0), \alpha \in\{\mathrm{B} 800, \mathrm{~B} 850\}$, are plotted in Fig. 5. $C_{\alpha}(0)=\left\langle\delta E^{2}\right\rangle$ represents the variance of the energy gap fluctuations with $C_{\mathrm{B} 800}(0)=3.16 \times 10^{-3} \mathrm{eV}^{2}$ and $C_{\mathrm{B} 850}(0)=8.68$ $\times 10^{-4} \mathrm{eV}^{2}$. The behavior of the two correlation functions is rather similar during the first 150 fs. Following a sharp decay to negative values in the first $9 \mathrm{fs}$, both functions exhibit an oscillatory component of approximately 18.5 fs period and uneven amplitudes that, in general, are larger for the B800. After $\sim 150 \mathrm{fs}$, the autocorrelation functions behave in a distinctive manner, both becoming negligibly small for $t \gtrsim 400$ fs.

The spectral densities $J_{\alpha}(\omega)$ for B800 and B850, determined according to Eq. (12), are shown in Fig. 6. The prominent peak about $\omega_{p}=0.22 \mathrm{eV}$ is due to the persistent $18.5 \mathrm{fs}$ oscillations in $C_{\alpha}(t)$. Being reported in previous studies, ${ }^{16,28}$ by using both ab initio (HF/CIS with STO-3G* basis set) and semiempirical QC methods, these spectral features appear to be intrinsic properties of BChl-a, most likely originating from a strong coupling of the pigment to intramolecular vibronic modes (e.g, $\mathrm{C}=\mathrm{O}$ or methine bridge stretching modes). The precise identification of the origin of these vibrations would require a detailed normal mode analysis of

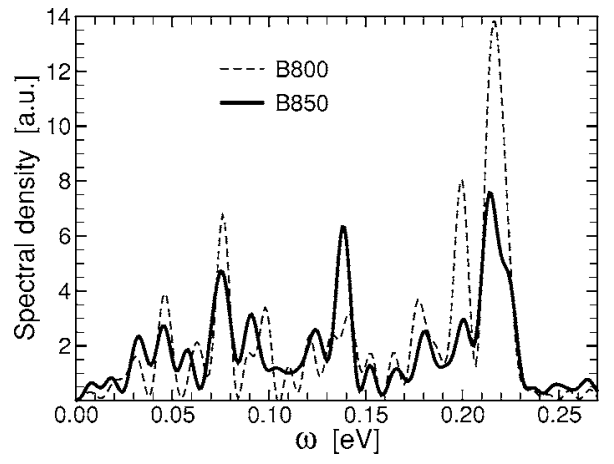

FIG. 6. Spectral density function $J(\omega)$ for B800 (dashed line) and B850 (solid line) obtained according to Eq. (12).

the PPC which is beyond the scope of the present work. Often, the environment in a PPC is modeled as an equivalent harmonic (phonon) heat bath for which the cumulant approximation is exact. ${ }^{3}$ The corresponding phonon spectral density can be written as $J(\omega)=\pi \omega^{2} \Sigma_{\lambda} g_{\lambda}^{2} \delta\left(\omega-\omega_{\lambda}\right)$, where $g_{\lambda}$ is the coupling constant to the phonon mode $\lambda$. Thus, one can interpret the magnitude of the spectral functions in Fig. 6 as a measure of the coupling strength to phonons of that particular frequency. The complex structure of the spectral functions indicates that all inter- and intramolecular vibronic modes with frequency below $\omega_{p}$ will contribute to the line shape function. Hence, attempts to use simplified model spectral functions appear to be unrealistic even if these may lead to absorption spectra that match the experimental results.

The line shape functions of individual B800 and B850, calculated from Eqs. (14), are plotted in Fig. 7. The origin of the frequency axis corresponds to the mean energy gaps $\omega_{\mathrm{B} 800}$ and $\omega_{\mathrm{B} 850}$, respectively. The highly polarized surrounding of the B800 BChls in Rs. molischianum [Fig. 1(b)] renders $A_{\mathrm{B} 800}(\omega)$ twice as broad $(\mathrm{FWHM} \approx 26 \mathrm{meV})$ as $A_{\mathrm{B} 850}(\omega)(\mathrm{FWHM} \approx 13 \mathrm{meV})$. Also, the redshift of the peak of the former $(\Delta \omega \approx 25 \mathrm{meV})$ is more than three times larger than that of the latter $(\Delta \omega \approx 7 \mathrm{meV})$.

Because at room temperature high frequency vibronic modes (with $\omega \gg 26 \mathrm{meV}$ ) are virtually unpopulated one may argue that in spite of the strong coupling of the $Q_{y}$ excitations to such modes [reflected by prominent peaks in the spectral function, e.g., the one about $\omega_{p}=0.22 \mathrm{eV}$ corresponding to the persistent oscillations of $\sim 18.5 \mathrm{fs}$ in $\left.C_{\alpha}(t)\right]$,

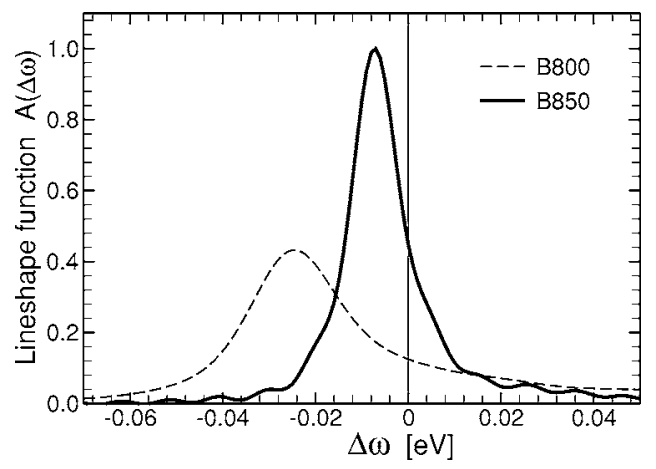

FIG. 7. Line shape functions $\bar{A}_{\mathrm{B} 800}(\Delta \omega)$ (dashed line) and $\bar{A}_{\mathrm{B} 850}(\Delta \omega)$ (solid line). 


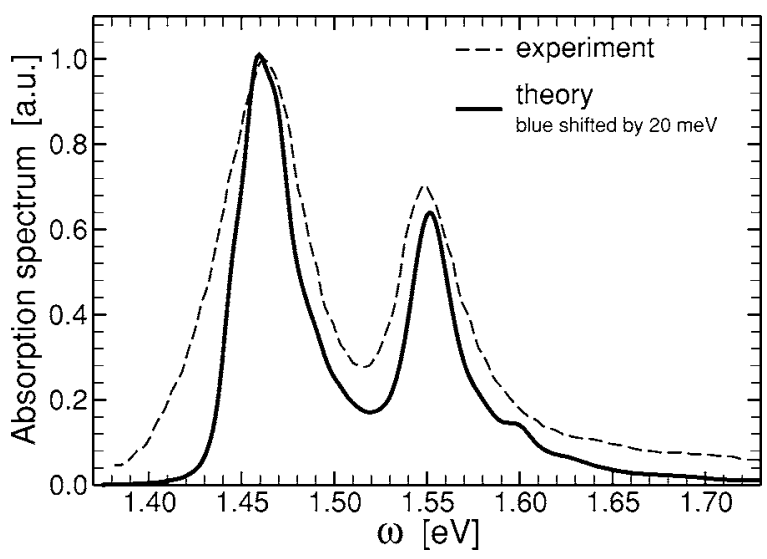

FIG. 8. Computed (solid line) and experimental (dashed line) absorption spectra (in arbitrary units) of the BChl aggregate in Rs. Molischianum LH2. The computed spectrum has been blueshifted by $20 \mathrm{meV}$ for best match.

these cannot have important effect on the line shape function and the corresponding OD spectrum. Indeed, the artificial removal of the $\omega_{p}$ peak in $J_{\alpha}(\omega)$ does not alter the recalculated line shape functions $\bar{A}_{\alpha}(\Delta \omega)$ apart from a slight reduction in the redshift of their peaks, i.e., by $\sim 6 \mathrm{meV}$ for B800 and $\sim 1 \mathrm{meV}$ for B850 (results not shown). We emphasize, however, that although in this case the omission of the high frequency end in the spectral functions would only cause a $\sim 5 \mathrm{meV}$ decrease in the $\sim 91 \mathrm{meV}$ separation between the B800 and B850 peaks in the OD spectrum $I(\omega)$ (see Fig. 8), in general the calculation of $I(\omega)$ requires the entire frequency range of the spectral functions.

Since the available simulation data are not sufficient to properly estimate the excitonic line shape functions $A_{J}(\omega)$, by neglecting the effect of exchange narrowing, ${ }^{14,27}$ we approximated these with $A_{\mathrm{B} 850}(\omega)$. Thus, the OD spectrum of the LH2 BChls was calculated by using the formula

$$
\begin{aligned}
I(\omega) \propto & \omega\left[\sum_{J} d_{J}^{2} \bar{A}_{\mathrm{B} 850}\left(\omega-\omega_{J}\right)\right. \\
& \left.+8 d_{\mathrm{B} 800}^{2} \bar{A}_{\mathrm{B} 800}\left(\omega-\omega_{\mathrm{B} 800}\right)\right],
\end{aligned}
$$

where $\omega_{J}=\left\langle\Delta E_{J}\right\rangle$.

As shown in Fig. 8, after an overall blueshift of $20 \mathrm{meV}$, $I(\omega)$ matches remarkably well the experimental OD spectrum, especially if we take into account that it was obtained from the sole knowledge of the high resolution crystal structure of LH2 from Rs. molischianum. The reason why both B800 and B850 peaks of $I(\omega)$ are somewhat narrower than the experimental ones is most likely due to the fact that the effect of static disorder is ignored in the present study. Indeed, our calculations were based on a single LH2 ring, while the experimental data are averaged over a large number of such rings. While computationally expensive, in principle, the effect of static disorder could be taken into account by repeating the above calculations for different initial configurations of the $\mathrm{LH} 2$ ring and then averaging the corresponding OD spectra.

To conclude this section we would like to relate the present work to previous two combined MD/QC studies. ${ }^{16,28}$ In Mercer et $a l^{28}$ it is argued that the $a b$ initio QC method
(HF/CIS with the STO-3G* basis set) should be preferred to semiempirical methods for calculating optical spectra because it reproduces better their experimental results. The FWHM of their calculated semiempirical and $a b$ initio absorption spectra of BChl-a in methanol are $\sim 65$ and $\sim 125 \mathrm{meV}$, respectively. These values are similar to the ones we obtained for the same type of calculations for BChl-a in vacuum and in LH2 embedded in its native environment. Since, except Ref. 28, all experimental results on the $Q_{y}$ absorption band of BChls we are aware of have a FWHM of $\$ 80 \mathrm{meV}$ at room temperature, we conclude that in fact the semiempirical ZINDO/S method should be preferable to the $a b$ initio QC method. In general, the latter overestimates the broadening of the OD spectrum by a factor of 2-3. In Damjanovic et al., ${ }^{16}$ the OD spectrum of individual B850s (i.e., without excitonic coupling) calculated with the $a b$ initio method yielded the same FWHM of $\sim 125 \mathrm{meV}$ as in Mercer et al. ${ }^{28}$ Once the excitonic coupling was included within the framework of a polaron model, and it was assumed that the entire oscillator strength was carried by a single exciton level of a perfect B850 ring, the FWHM of the resulting OD spectrum was reduced to $\sim 43 \mathrm{meV}$ as a result of exchange narrowing. Thus the obtained OD spectrum for the B850s appeared to match rather well the corresponding part of the experimental one. However, by applying the same method to the B800s, where there is no exchange narrowing, the polar environment further broadens the corresponding OD spectrum to a FWHM of $\sim 250 \mathrm{meV}$ that is clearly unphysically large. Thus, the conclusion again is that the ZINDO/S CIS semiempirical method should be preferred for calculating optical spectra of PPCs.

\section{Circular dichroism (CD) spectrum}

The CD spectrum of the LH2 BChls from Rs. molischianum was determined by following the theoretical approach described at the end of Sec. II and by employing the same time series (obtained from the combined MD/QC calculations) used for calculating the OD spectrum.

First, the rotational strengths of both B850 excitons and B800 BChls were determined by using Eq. (22b). In this equation, just like in the case of the point-dipole interaction matrix elements (15), the vector $\mathbf{r}_{n}$ described the position of the $\mathrm{Mg}$ atom in the $n$th BChl. As already clarified in Sec. II the calculation of the rotational strength of the B800 BChls requires solving the corresponding excitonic Hamiltonian (16) regardless of how small the dipole-dipole coupling is between these BChls. The calculation does not yield either noticeable corrections to the B800 excitation energies or admixture of the corresponding $Q_{y}$ states, however, it leads to sizable mean rotational strengths as shown in Fig. 9 (filled circles). Similarly to the TDM strengths (Fig. 3), the largest (negative) mean rotational strengths are carried by the four lowest B850 excitonic states as shown in Fig. 9 (open squares). The second highest excitonic state also has a sizable rotational strength and is responsible for enhancing the positive peak of the B800 contribution to the CD spectrum [Fig. 10(a)].

Second, the CD spectrum is calculated from Eq. (22a) 


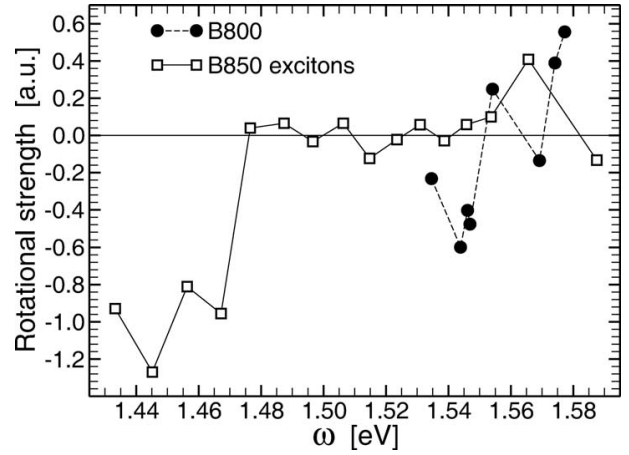

FIG. 9. Mean rotational strength of the excitonically coupled B800 (circles) and B850 (rectangles) BChls as a function of the corresponding excitonic energies. The purpose of the thin lines are to guide the eyes.

where the summation index $J$ runs over all B850 and B800 excitonic states and $A_{J}(\omega)=\bar{A}_{\alpha}\left(\omega-\omega_{J}\right)$, with $\alpha$ $\in\{\mathrm{B} 850, \mathrm{~B} 800\}$. Figure 10(a) shows the CD spectrum contribution by the B850 (solid line) and B800 (dashed line). Both contributions have the same qualitative structure with increasing energy: a pronounced negative peak followed by a smaller positive one. The B850 negative CD peak is about twice as large as the corresponding B 800 peak. The total $C D$ spectrum, given by the superposition of the B850 and B800 contributions, is shown in Fig. 10(b) (solid line) and matches fairly well the experimental spectrum $^{19}$ (dashed line). It should be emphasized that apart from an overall scaling factor the $\mathrm{CD}$ spectrum was calculated from the same MD/QC data as the OD spectrum by following the procedure described above.

\section{CONCLUSIONS}

The continuous increase in processor power and availability of high performance computer clusters, along with the growing number of high resolution crystal structures of membrane bound pigment-protein complexes, make feasible the theoretical characterization of the spectral and optical properties of such systems at atomic level. By applying an approach that combines all atom MD simulations, efficient

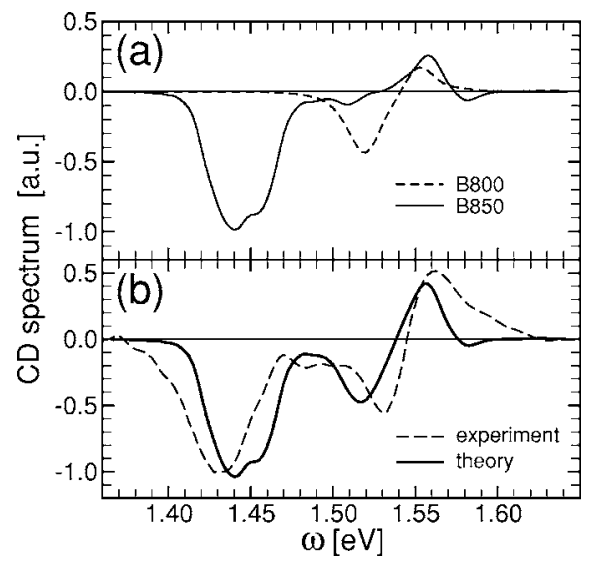

FIG. 10. (a) CD spectrum contributions due to B800 (dashed line) and B850 (solid line) BChls. (b) Comparison between the computed (solid line) and experimental CD spectra of the BChl aggregate in Rs. Molischianum LH2. semiempirical QC calculations, and quantum many-body theory, we have shown that starting solely from the atomic structure of the LH2 ring from Rs. molischianum the OD and CD spectra of this PPC can be predicted with reasonable accuracy at affordable computational costs. The configuration snapshots taken with femtosecond frequency during the MD simulation of the PPC in its native, fully solvated lipidmembrane environment at room temperature and normal pressure provide the necessary input for the QC calculations of the optical excitation energies and transition dipole moments of the pigment molecules. The obtained time series are used to evaluate within the second cumulant approximation the optical line shape functions as the Fourier transform of the quantum dipole-dipole correlation function. Our choice of the ZINDO/S CIS method for the QC calculations was motivated by the fact that it is almost two orders of magnitude faster and much more accurate than the most affordable $a b$ initio method (HF/CIS with the STO- $3 \mathrm{G}^{*}$ basis set). Compared to the former, the latter method overestimates by a factor of 2-3 both the excitation energies and the broadening of the energy spectrum. Just like in several previous studies, ${ }^{19,20,45,46}$ we have found that the ZINDO/S method repeatedly yields results in good agreement with existing experimental data.

By investigating the excitation energy spectrum of the LH2 BChls both in the presence and in the absence of the atomic partial charges of their environment, we have convincingly demonstrated that the large broadening of the B800 peak is due primarily to the electric field fluctuations created by the polar surrounding environment of the B800s. There is no such effect for the B850s which sit in a nonpolar local environment. The broadening of the B850 peak is due to the sizable excitonic coupling between these BChls. Since only the lowest three excitonic states carry most of the available dipole strength, in spite of the $\sim 0.2 \mathrm{eV}$ wide excitonic band, the B850 absorption peak has a FWHM only slightly larger than the B800 one.

It is rather remarkable that both the OD and the CD spectra of the considered LH2 complex are fairly well predicted by our combined MD/QC method. However, a more thorough testing of the proposed method, involving other PPCs, is necessary to fully establish its capability of predicting optical properties by using only atomic structure information.

\section{ACKNOWLEDGMENTS}

This work was supported in part by grants from the University of Missouri Research Board, the Institute for Theoretical Sciences, a joint institute of Notre Dame University and Argonne National Laboratory, the U.S. Department of Energy, Office of Science through Contract No. W-31-109ENG-38, and NSF through Grant No. FIBR-0526854. One of the authors (A.D.) acknowledges support from the Burroughs Welcome Fund. The authors also acknowledge computer time provided by NCSA Allocations Board grant MCB020036. 
${ }^{1}$ T. Renger, V. May, and O. Kuhn, Phys. Rep., Phys. Lett. 343, 138 (2001).

${ }^{2}$ V. Chernyak, W. M. Zhang, and S. Mukamel, J. Chem. Phys. 109, 9587 (1998).

${ }^{3}$ S. Mukamel, Principles of Nonlinear Optical Spectroscopy (Oxford University Press, New York, 1995).

${ }^{4}$ V. May and O. Kühn, Charge and Energy Transfer Dynamics in Molecular Systems (Wiley-VCH, Berlin, 2000).

${ }^{5} \mathrm{H}$. van Amerongen, L. Valkunas, and R. van Grondelle, Photosynthetic Excitons (World Scientific, Singapore, 2000).

${ }^{6}$ J. Koepke, X. C. Hu, C. Muenke, K. Schulten, and H. Michel, Structure (London) 4, 581 (1996).

${ }^{7}$ G. McDermott, S. Prince, A. Freer, A. Hawthornthwaite-Lawless, M. Papiz, R. Cogdell, and N. Isaacs, Nature (London) 374, 517 (1995).

${ }^{8}$ X. Hu, T. Ritz, A. Damjanovic, F. Autenrieth, and K. Schulten, Q. Rev. Biophys. 35, 1 (2002).

${ }^{9}$ M. Yang, R. Agarwal, and G. R. Fleming, J. Photochem. Photobiol., A 142, 107 (2001).

${ }^{10}$ V. Sundstrom, T. Pullerits, and R. van Grondelle, J. Phys. Chem. B 103, 2327 (1999).

${ }^{11}$ H. M. Wu, M. Ratsep, R. Jankowiak, R. J. Cogdell, and G. J. Small, J. Phys. Chem. B 101, 7641 (1997).

${ }^{12}$ L. M. P. Beekman, R. N. Frese, G. J. S. Fowler, R. Picorel, R. J. Cogdell, I. H. M. vanStokkum, C. N. Hunter, and R. van Grondelle, J. Phys. Chem. B 101, 7293 (1997).

${ }^{13}$ S. Georgakopoulou, R. N. Frese, E. Johnson, C. Koolhaas, R. J. Cogdell, R. van Grondelle, and G. van der Zwan, Biophys. J. 82, 2184 (2002).

${ }^{14}$ O. J. G. Somsen, R. van Grondelle, and H. van Amerongen, Biophys. J. 71, 1934 (1996).

${ }^{15}$ G. D. Scholes and G. R. Fleming, J. Phys. Chem. B 104, 1854 (2000).

${ }^{16}$ A. Damjanovic, I. Kosztin, U. Kleinekathofer, and K. Schulten, Phys. Rev. E 65, 031919 (2002).

${ }^{17}$ Z. He, V. Sundstrom, and T. Pullerits, J. Phys. Chem. B 106, 11606 (2002).

${ }^{18}$ X. Hu, A. Damjanovic, T. Ritz, and K. Schulten, Proc. Natl. Acad. Sci U.S.A. 95, 5935 (1998a).

${ }^{19}$ J. A. Ihalainen, J. Linnanto, P. Myllyperkio, I. H. M. van Stokkum, B. Ucker, H. Scheer, and J. E. I. Korppi-Tommola, J. Phys. Chem. B 105, 9849 (2001)

${ }^{20}$ J. Linnanto, J. E. I. Korppi-Tommopa, and V. M. Helenius, J. Phys. Chem. B 103, 8739 (1999).

${ }^{21}$ T. Meier, Y. Zhao, V. Chernyak, and S. Mukamel, J. Chem. Phys. 107, 3876 (1997).

${ }^{22}$ J. Ray and N. Makri, J. Phys. Chem. A 103, 9417 (1999).

${ }^{23}$ S. J. Jang and R. J. Silbey, J. Chem. Phys. 118, 9324 (2003).

${ }^{24}$ X. Hu and K. Schulten, Phys. Today 50(8), 28 (1997).
${ }^{25}$ X. Hu, A. Damjanović, T. Ritz, and K. Schulten, Proc. Natl. Acad. Sci. U.S.A. 95, 5935 (1998).

${ }^{26}$ J.-P. Zhang, R. Fujii, P. Qian, T. Inaba, T. Mizoguchi, and Y. Koyama, J. Phys. Chem. B 104, 3683 (2000).

${ }^{27}$ H. van Amerongen, L. Valkunas, and R. van Grondelle, Photosynthetic Excitons (World Scientific, Singapore 2000).

${ }^{28}$ I. Mercer, I. Gould, and D. Klug, J. Phys. Chem. B 103, 7720 (1999).

${ }^{29}$ V. May and O. Kühn, Charge and Energy Transfer Dynamics in Molecular Systems (Wiley-VCH, Berlin, 2000).

${ }^{30}$ N. Makri, J. Phys. Chem. B 103, 2823 (1999).

${ }^{31}$ K. Schulten and M. Tesch, Chem. Phys. 158, 421 (1991).

${ }^{32}$ S. A. Egorov, K. F. Everitt, and J. L. Skinner, J. Phys. Chem. A 103, 9494 (1999)

${ }^{33}$ W. Humphrey, A. Dalke, and K. Schulten, J. Mol. Graphics 14, 33 (1996).

${ }^{34}$ A. D. MacKerell, Jr., D. Bashford, M. Bellott et al., FASEB J. 6(1), A143 (1992).

${ }^{35}$ A. D. MacKerell, Jr., D. Bashford, M. Bellott et al., J. Phys. Chem. B 102, 3586 (1998)

${ }^{36}$ M. Schlenkrich, J. Brickmann, A. D. MacKerell, Jr., and M. Karplus, in Biological Membranes: A Molecular Perspective from Computation and Experiment, edited by K. M. Merz and B. Roux (Birkhauser, Boston, 1996), pp. 31-81.

${ }^{37}$ W. L. Jorgensen, J. Chandrasekhar, J. D. Madura, R. W. Impey, and M. L. Klein, J. Chem. Phys. 79, 926 (1983).

${ }^{38}$ S. E. Feller, Y. H. Zhang, R. W. Pastor, and B. R. Brooks, J. Chem. Phys. 103, 4613 (1995).

${ }^{39}$ T. Darden, D. York, and L. Pedersen, J. Chem. Phys. 98, 10089 (1993).

${ }^{40}$ J. C. Phillips, R. Braun, W. Wang, J. Gumbart, E. Tajkhorshid, E. Villa, C. Chipot, R. D. Skeel, L. Kale, and K. Schulten, J. Comput. Chem. 26, 1781 (2005).

${ }^{41}$ S. Miyamoto and P. A. Kollman, J. Comput. Chem. 13, 952 (1992).

${ }^{42}$ M. G. Cory, M. C. Zerner, X. Hu, and K. Schulten, J. Phys. Chem. B 102, 7640 (1998)

${ }^{43}$ J. Ridley and M. Zerner, Theor. Chim. Acta 32, 111 (1973).

${ }^{44}$ M. Zerner, G. Loew, R. Kirchner, and U. J. Mueller-Westerhoff, J. Am. Chem. Soc. 102, 589 (1980).

${ }^{45}$ J. Linnanto and J. Korppi-Tommola, J. Comput. Chem. 25, 123 (2004).

${ }^{46}$ A. Damjanovic, H. M. Vaswani, P. Fromme, and G. R. Fleming, J. Phys. Chem. B 106, 10251 (2002b).

${ }^{47}$ HYPERCHEM $^{\mathrm{TM}}$, Hypercube, Inc., 1115 NW 4th Street, Gainesville, Florida 32601

${ }^{48}$ M. J. Frisch, G. W. Trucks, H. B. Schlegel et al., GAUSSIAN 98, Gaussian Inc., Pittsburgh, PA, 1998.

${ }^{49}$ K. Visscher, H. Bergstrom, V. Sundström, C. Hunter, and R. van Grondelle, Photosynth. Res. 22(3), 211 (1989). 